\title{
Immunotherapies for Pediatric Solid Tumors: A Targeted Update
}

\author{
Ajay Gupta ${ }^{1}$ (D) Timothy P. Cripe ${ }^{2}$ (I) \\ Accepted: 29 October 2021 / Published online: 25 November 2021 \\ (c) The Author(s), under exclusive licence to Springer Nature Switzerland AG 2021
}

\begin{abstract}
There are encouraging signs in our collective progress to leverage the immune system to treat pediatric cancers. Here, we summarize interim successes in cancer immunotherapy and opportunities to translate from the adult world to pediatrics, and highlight challenges that could benefit from additional development, focusing on solid tumors. Just a decade ago, other than antibodies targeting disialoganglioside (GD2) in neuroblastoma, pediatric cancer immunotherapy was mostly relegated to obscure preclinical studies in a few academic labs. Today there are numerous clinical trials of a variety of antibody, cellular, gene, and viral therapies and vaccines designed to either promote antitumor immunity or specifically attack validated immunotherapy targets. Understanding those targets and their pediatric relevance is paramount. While much work is underway to evaluate the utility of numerous immunologic targets, the lack of regulatory approvals is emblematic of the challenges that remain. Herein we focus our review on the most promising targeted immunotherapies in clinical trials for children.
\end{abstract}

\section{Key Points}

Treatment of pediatric solid tumors is starting to benefit from a variety of cell-surface targets and new approaches to attack those targets.

The most promising of these targets include: immune checkpoints, GD2, B7-H3, HER2, and CD47.

Future directions will likely include combinations of therapies, biomarkers to assess success, the creation of more antibody-drug conjugates, and further breakdown of regulatory barriers.
Ajay Gupta

ajay.gupta@roswellpark.org

1 Division of Pediatric Oncology, Roswell Park Comprehensive Cancer Center, Department of Pediatrics, University at Buffalo Jacobs School of Medicine and Biomedical Sciences, Buffalo, New York, USA

2 Division of Hematology/Oncology/Blood and Marrow Transplantation, Department of Pediatrics, Nationwide Children's Hospital, The Ohio State University, Columbus, Ohio, USA

\section{Introduction}

Since our (TPC) initial review of the pediatric cancer immunotherapy landscape three years ago [1], cancer immunotherapy has undergone significant advances for both pediatric and adult patients. There are some encouraging signs in our collective progress to leverage the immune system to treat pediatric cancers just over the past few years. Here we focus on new successes and persistent challenges in pediatric solid tumors since advances in leukemia/lymphoma have been recently addressed elsewhere [2, 3]. Because the array of therapeutics and strategies have become so broad, we mainly highlight selected efforts that have reached the clinic (Table 1).

Different aspects of the immune system recognize different targets. Generally speaking, antibodies can bind a wide variety of antigens, ranging from proteins and peptides to carbohydrates [4], whereas native $T$ cells recognize peptides presented in the context of the class I major histocompatibility complex (MHC). NK cells can recognize antibodycoated cells via their Fc receptor, or directly interact with stress molecules on cell surfaces [5]. In addition, both $\mathrm{T}$ and NK cells have been modified by gene transfer or CRISPR/ Cas technologies to be endowed with molecularly engineered cell surface receptors that redirect them to specific targets, so understanding those targets and their pediatric relevance is paramount. In this review, we have chosen to specifically highlight novel targeted immunotherapies in 


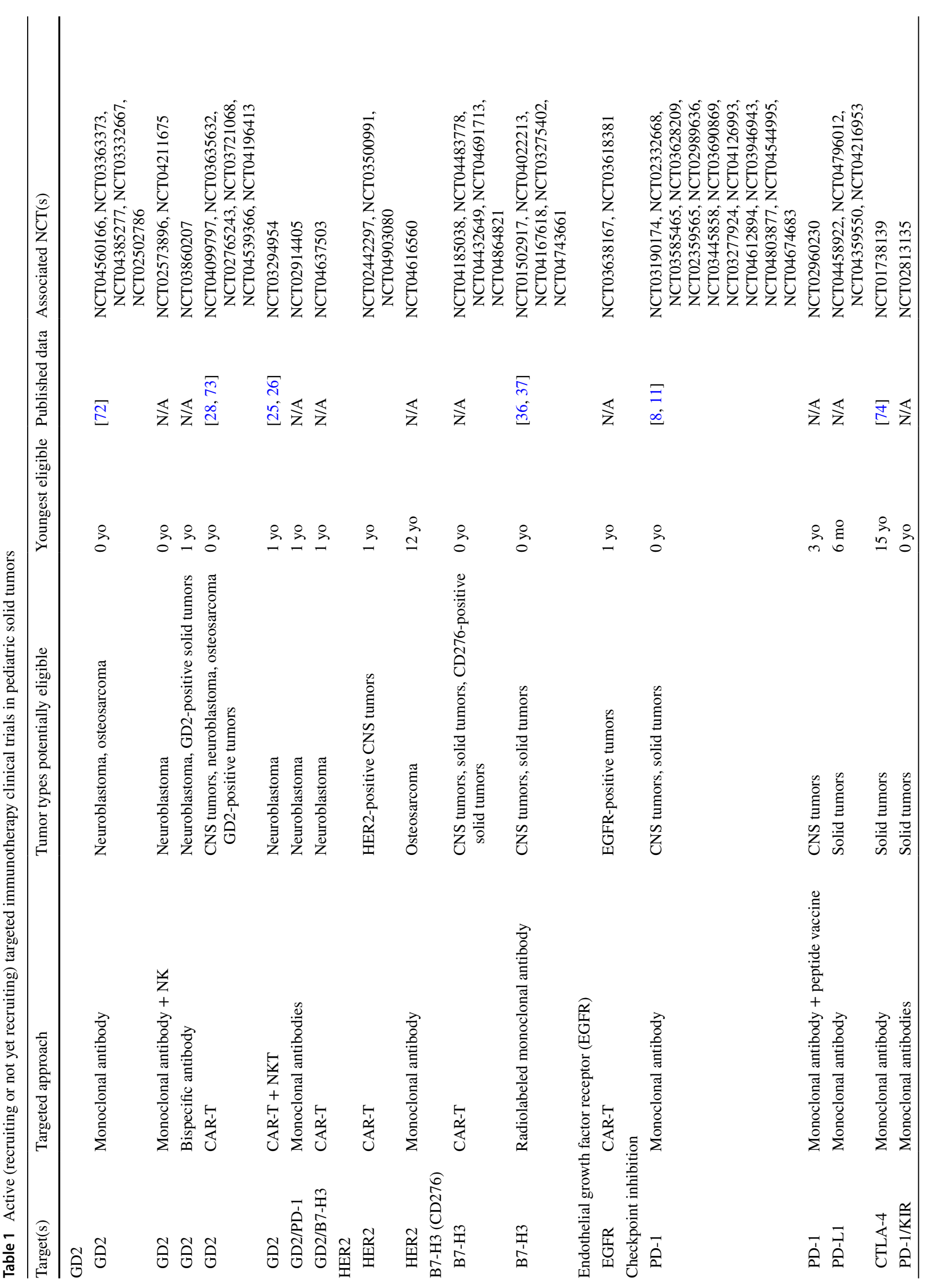




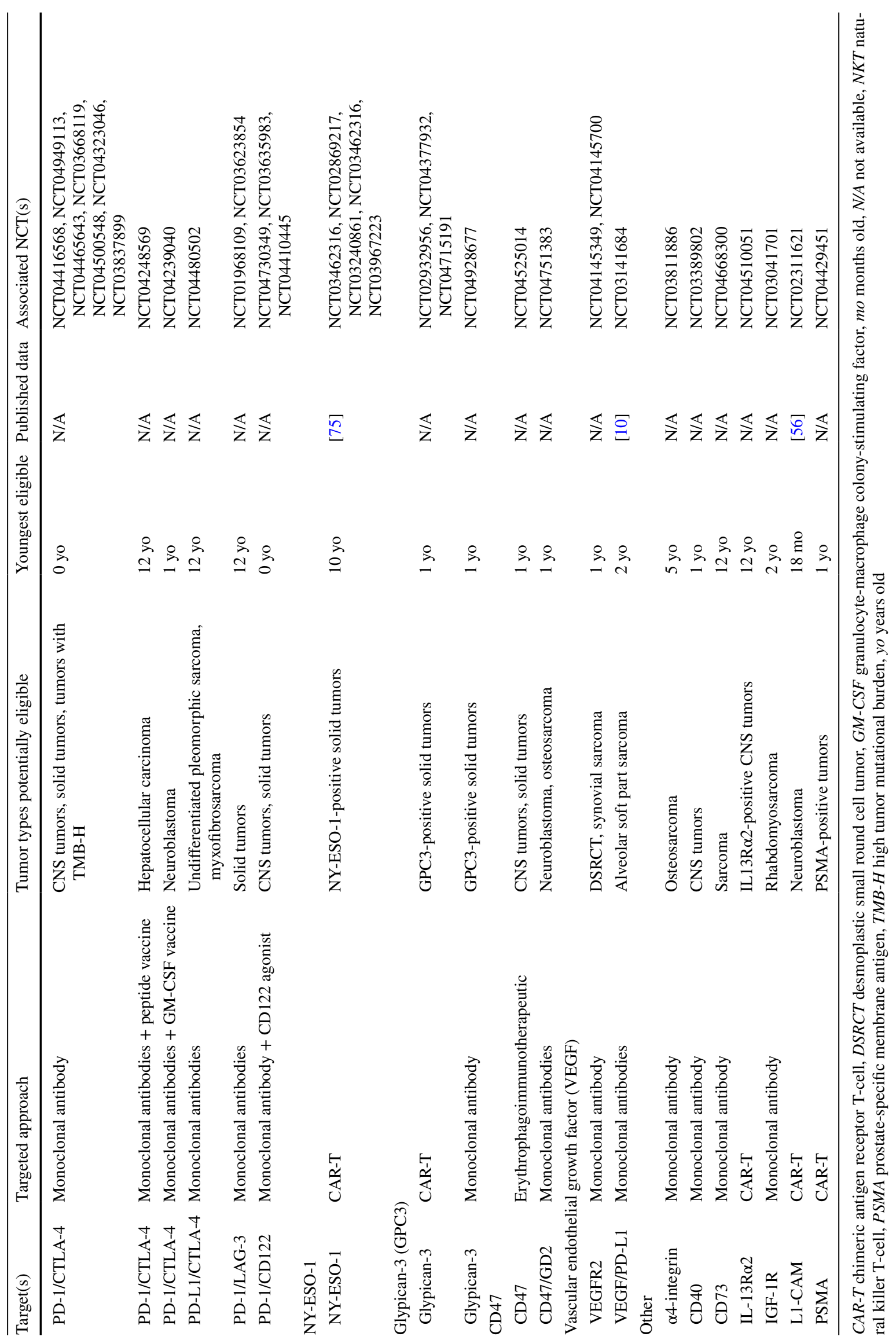


pediatric solid tumors, with brief mention of non-targeted immunotherapies.

\section{Immunotherapeutic Targets}

We summarize reported data in pediatric solid tumors to date, splitting the section into those targets that have been extensively researched and those that require further characterization.

\subsection{Targets with Extensive Research (Arranged by Priority)}

\subsubsection{Immune Checkpoint Inhibitors (PD-1, PD-L1, CTLA-4)}

The successes in selected adult cancers of blocking immune checkpoints such as PD1 rely on an underlying target that can be recognized by immune executioner cells. Indeed, these agents have been approved for pediatric patients with high mutational rates, such as those with inherited mismatch repair deficiency [6]. Yet such mutations are not the norm in pediatric cancers. The Children's Oncology Group (COG) phase I/II trial ADVL1412 was reported on patients aged 1-30 years with relapsed/refractory solid tumors treated with single-agent nivolumab [7]. Seventy-five patients were evaluable for toxicity and the drug was found to be safe and well tolerated with a recommended phase II dose established; efficacy was only shown in $20 \%$ of patients with lymphoma (4 of 20; 1 complete response [CR] and 3 partial responses $[\mathrm{PR}]$ ). Based on no objective responses in patients with solid tumors, it was suggested that the agent only be studied in combination with other immunotherapies in pediatric histologies. In addition, in the KEYNOTE-051 study, investigators treated 154 pediatric patients aged 6 months to 17 years with melanoma or PD-L1-positive relapsed/refractory solid tumors or lymphoma with single-agent pembrolizumab. Again, while generally well tolerated, the majority of responses were seen in patients with lymphomas; patients with solid tumors experienced a $5.9 \%$ objective response overall [8]. A third study (SARC028) assessed the benefit of pembrolizumab in a phase II trial that included a cohort of pediatric patients 12 years and older with bone sarcomas [9]. Out of the 40 patients with bone sarcomas, it was not specified what percentage were pediatric; however, there were only two objective responses, one in a patient with osteosarcoma (with ongoing benefit; 1 of 22 or $4.5 \%$ ) and one in a patient with chondrosarcoma ( 1 of 5 or $20 \%$ ). Finally, investigators treating 43 patients with advanced alveolar soft part sarcoma on a phase II study with atezolizumab, a PD-L1 antibody, demonstrated impressive responses with one CR and 15 PR (16/43 or 37\%) and good tolerability with seven grade 3 events reported (16\%) [10].
A few trials have reported on checkpoint blockade in combination therapy. One group combined nivolumab with nab-sirolimus, an mTOR inhibitor, in nine adult patients with advanced sarcomas and observed no dose-limiting toxicities (DLTs); on the third dose level, the median progression-free survival had not yet been reached at the time of the conference report, though the follow-up time was not specified [11]. A European multicenter group tested 13 children aged 5-19 years with relapsed/refractory tumors with a basket trial, one arm of which assessed nivolumab with metronomic cyclophosphamide plus or minus radiation in 13 patients and found it well tolerated with an unconfirmed PR in a patient with desmoplastic small round cell tumor (DSRCT) $(1 / 3$ or $33 \%)$ and a patient with ependymoma (1/1) [12]. A different arm is currently open and assessing nivolumab plus lirilumab, an anti-Killer Ig-like receptor (KIR) antibody designed to upregulate NK cell activation (ClinicalTrials.gov identifier: NCT02813135).

One potential limitation of immune checkpoint inhibition is that pediatric solid tumors exhibit low immunogenicity due to low mutational burden and/or downregulation of MHC-I expression, leading to low tumor infiltration of activated T and NK cells. Multiple publications have demonstrated the variable proliferation of these tumor infiltrating lymphocytes (TILs) in pediatric histologies (and significant variation between individuals) including in neuroblastoma [13], Wilms tumor [14], germ cell tumors [15], brain tumors [16], and osteosarcoma [17]. Interestingly, the degree of tumor cell differentiation in neuroblastoma appears to correlate with degree of TIL infiltration as well as increased MHC-I expression and improved outcome [13]. The mechanism and purpose of low MHC-I expression may be manifold; low MHC-I expression may simply be a marker of undifferentiated (and therefore more aggressive) tumor cells [13] or tumor cells may actively downregulate MHC-I expression to avoid T-cell recognition. Dysregulation of MHC-I expression in cancer has been reviewed elsewhere [18].

\subsubsection{Disialoganglioside GD2}

Gangliosides, including GD2 and GD3, are cell surface markers that are expressed in high levels on specific tumors, most commonly neuroblastoma and osteosarcoma. Investigators have developed multiple anti-GD2 chimeric monoclonal antibodies (including dinutuximab, humanized 3F8 [Hu3F8], hu14.18K322A) and extensively studied each in neuroblastoma. A recent update from a phase III trial (HR-NBL1/SIOPEN) demonstrated no additional benefit and added toxicity with interleukin (IL)-2 when given with dinutuximab to patients with high-risk neuroblastoma [19], leading to discontinuation of use of IL-2 in similar COG 
protocols. Other recent advances with anti-GD2 antibodies in neuroblastoma are reviewed elsewhere [20].

Osteosarcoma has shown to have $>95 \%$ GD2 positivity in tumors and cell lines [21] and similar efforts have been made to emulate the progress of anti-GD2 therapy in neuroblastoma. In the COG study AOST1421, investigators studied dinutuximab in combination with granulocyte-macrophage colony-stimulating factor (GM-CSF) in patients with recurrent pulmonary osteosarcoma in complete surgical remission and enrolled 39 evaluable patients with a 12-month eventfree survival (EFS) of 30\%, an insignificant improvement over a historical 12-month EFS of 20\% [22]. There is an ongoing phase II study of Hu3F8 plus GM-CSF in the treatment of recurrent osteosarcoma in second remission or later (NCT02502786). In a phase I setting, the National Cancer Institute is set to assess the combination of dinutuximab plus magrolimab (monoclonal antibody against CD47, the so-called macrophage 'don't eat me signal,' see below and reviewed here [23]) in relapsed/refractory neuroblastoma or relapsed osteosarcoma (NCT04751383). Additional GD2 research has been recently summarized [24] and addresses future possibilities secondary to GD2 positivity in pediatric melanoma, Ewing sarcoma, and retinoblastoma.

GD2 has also been targeted via chimeric antigen receptor (CAR) modification of both T cells and NKT cells [25], which are innate-like $\mathrm{T}$ cells that acquire NK-like properties in the thymus and normally only recognize CD1d. Clinicians conducting a phase I trial gave 11 patients with relapsed/refractory neuroblastoma CAR-NKTs and demonstrated safety and two objective responses (18\%, $1 \mathrm{CR}$ and 1 PR) [26]. Recently, as reported in an abstract from Cancer Research UK Phase I trials of anti-GD2 CAR-T cells (1RGCART), investigators treated 12 patients with relapsed/ refractory neuroblastoma with no DLTs, although there was also no persistence of the CARs until dose level $4\left(1 \times 10^{8}\right.$ cells per $\mathrm{m}^{2}$ ) [27]. Unfortunately, all patients developed progressive disease and further dose escalation trials have been proposed. A Chinese study with 34 high-risk neuroblastoma patients and positive GD2 expression assessed consolidative treatment with a second-generation CAR-T against GD2 (4SCAR-GD2) after surgery, chemotherapy, and radiation. Investigators found that the CAR-T cells were well tolerated with no grade 3 or 4 toxicities observed, exhibited extended CAR persistence, and demonstrated PR in five patients including two patients with marked tumor regression [28]. Additional efforts are reviewed by Richards et al. [29].

A German group combined anti-GD2 therapy with immune checkpoint inhibition in a case report of two patients with relapsed/refractory metastatic neuroblastoma treated with nivolumab and dinutuximab and achieving a $\mathrm{CR}$ and a very good partial response (VGPR) [30]. Others have combined third-generation GD2-specific CAR-T cells with pembrolizumab in a phase I trial that treated 11 patients with relapsed or refractory neuroblastoma [31]. While no objective responses were demonstrated while on the trial, two out of three patients were reported to have long-term outcomes of CR; these were the only three patients treated with the combination of CAR-T, a conditioning regimen, and nivolumab.

GD2-targeted therapy has also been combined with NK cells. In a phase II trial of humanized anti-GD2 monoclonal antibody (hu14.18K322A) plus haploidentical NK cells, IL-2, GM-CSF, and chemotherapy, investigators enrolled 13 heavily pre-treated patients with relapsed/refractory neuroblastoma. Four patients discontinued treatment for adverse events but there was a CR or PR in eight patients $(61.5 \%)$, demonstrating feasibility and clinically meaningful responses [32]. In a similar setting of patients with resistant neuroblastoma, a phase I/II trial tested a different anti-GD2 monoclonal antibody (m3F8) with increasing doses of haploidentical NK cells. Thirty-five patients were treated on five dose levels with no maximum tolerated dose (MTD) reached, and ten patients (29\%) experienced CR or PR to therapy [33]. A third group investigated the safety of haploidentical NK cells and IL-2 following haploidentical stem cell transplant and conditioning regimen of I-131 MIBG, cyclophosphamide, fludarabine, and ATG in seven children with recurrent neuroblastoma [34]. Unfortunately, six patients died, five of disease progression and one of treatment-related mortality. Another phase II trial assessed a novel conditioning regimen for newly diagnosed patients with high-risk neuroblastoma using busulfan, melphalan, autologous hematopoietic stem cell transplantation, hu14.18K322A, IL-2, and haploidentical NK cells [35]. Of the 30 treated patients, 21 received NK cells, which were well tolerated, but there was no clinical difference seen in patients who did or did not receive NK cells.

Given the successes of anti-GD2 therapy in neuroblastoma and its subsequent incorporation into first-line therapy, we think that other GD2-positive tumors such as osteosarcoma may benefit from GD2 combination therapy (whether with anti-CD47, checkpoint inhibitor, or NK cell therapies) as well as further CAR refinement.

\subsubsection{B7-H3 (CD276)}

B7-H3 is another immune checkpoint molecule that is overexpressed on multiple cancers. In a phase I trial of convection-enhanced delivery of radiolabeled antibody I-124-8H9 (anti-B7-H3) to pediatric patients with non-progressive diffuse midline gliomas (DMG), investigators enrolled 46 patients and found two DLTs and eight transient grade 3 toxicities with a median survival of 14.8 months, although four patients $(8.7 \%)$ had over 3 years' survival [36, 37]. In another phase I trial that evaluated intraperitoneal I-131-omburtamab (radiolabeled anti-B7-H3 monoclonal antibody) 
in 52 patients aged 1 year and older with DSRCT and other B7-H3-expressing tumors, investigators found no MTD or DLTs but two transient grade 4 neutropenias and one thrombocytopenia [38]. The therapy was also safely combined with whole abdominopelvic radiotherapy and showed a survival signal in both DSRCT patients and recurrent rhabdomyosarcoma to the peritoneal compartment; recently updated data showed an improvement in medial overall survival (OS) for 25 patients who received omburtamab plus whole abdomen radiation versus 30 patients who received radiation alone (median OS of 54 months versus 34 months, respectively) [39]. Researchers found even more impressive results when this drug was delivered intrathecally in a phase I trial to 80 treated patients with neuroblastoma metastatic to the central nervous system (CNS), where 45 (56\%) of the patients were alive at a median of 58 months of followup [40]. This drug is now being studied systemically in the same population in an open trial (NCT03275402). A third anti-B7-H3 antibody, MGA27, has reached the clinic in the form of a phase I trial for 24 patients aged 1-35 years with B7-H3-positive relapsed/refractory solid tumors, but has yet to report the results (NCT02982941).

Most recently, a promising new CAR-T has been developed called synthetic Notch (synNotch) CAR-T cell, which is able to recognize first GD2, and then upon recognition, activate a receptor to recognize $\mathrm{B} 7-\mathrm{H} 3$, both of which are expressed on neuroblastoma [41]. While this specific technology is in preclinical stages, there is a Chinese trial studying fourth-generation safety-designed CAR-T cells (4SCART) targeting multiple antigens, including GD2, B7-H3, and PSMA (prostate-specific membrane antigen) in neuroblastoma (NCT04637503).

Anti-B7-H3 therapy seems to be most effective in cancers with the worst overall up-front prognoses (DMG, DSRCT, neuroblastoma metastatic to the CNS). Multiple creative ways of delivering these antibodies appear to be effective in select individuals, though further studies to determine optimal dosing and routes are necessary. We are very interested to see how well the combination of GD2 and B7-H3 CAR will work.

\subsubsection{HER2}

HER2 is a well-known surface marker for multiple cancer types, most famously breast cancer. Autologous CAR-T cells against HER2 plus lymphodepleting chemotherapy in advanced HER2-positive pediatric sarcomas are being studied in an ongoing phase I trial (NCT00902044) in patients with refractory or metastatic sarcomas, with interim results from ten patients (median age 14 years) demonstrating safety and CR in two patients (20\%), one with osteosarcoma metastatic to the lungs and another in a patient with refractory alveolar rhabdomyosarcoma metastatic to bone marrow [42].
The latter patient had an exceptional response likely secondary to a revival of endogenous immune antitumor reactivity with disease remission for 20 months followed by a relapse that was again salvaged by a second infusion of the same CAR-T cells [43]. HER2 CAR-modified virus-specific T cells (VSTs) have also been studied in patients with progressive HER2-positive glioblastoma in a phase I trial that included seven pediatric patients and demonstrated one PR in a 17-year-old with an unresectable thalamic glioblastoma [44]. A COG Pediatric Early Phase Clinical Trial Network (PEP-CTN) trial, PEPN1924, is currently evaluating in phase II the use of an anti-HER2 monoclonal antibodydrug conjugate, trastuzumab deruxtecan, for the treatment of HER2-positive newly diagnosed or recurrent osteosarcoma (NCT04616560). We look forward to the results of this study and also expect to see additional explorations of existing CARs and VSTs.

\subsubsection{Vascular Endothelial Growth Factor (VEGF) and Receptor (VEGFR)}

VEGF is a key mediator of angiogenesis and tumor survival. Bevacizumab (anti-VEGF monoclonal antibody) was studied retrospectively in a pediatric cohort of 18 patients with relapsed/refractory sarcomas in combination with appropriate chemotherapeutic regimens and was shown to be generally well tolerated aside from cytopenias, and appeared to be associated with prolonged disease-free intervals in some patients with widely metastatic disease [45]. Investigators conducted a phase I study looking at the combination of bevacizumab with everolimus, an mTOR inhibitor, in 15 children with recurrent or refractory solid tumors and identified no DLTs and no objective responses [46]. A second phase I study evaluated the combination of bevacizumab, sorafenib, and low-dose cyclophosphamide in 24 children and found grade III/IV toxicities of lymphopenia in 17 patients, hypertension in four patients, hand/foot rash in three patients, and elevated lipase in three patients. Three patients achieved a PR, including two synovial sarcoma (2/3 or $66 \%)$ and one rhabdoid tumor patient (1/3 or 33\%) [47]. A European group conducted a randomized phase II study (BERNIE) evaluating the addition of bevacizumab to chemotherapy in children with metastatic rhabdomyosarcoma and non-rhabdomyosarcoma soft tissue sarcomas and found no difference in OS with the addition of bevacizumab $[48,49]$. Bevacizumab was used in a second phase II trial in addition to standard chemotherapy for 31 patients with osteosarcoma and demonstrated increased wound healing complications without any improvement in histologic response or survival [50]. The results from a recently completed phase I trial of ramucirumab (anti-VEGFR2) in 29 children aged 1-21 years with recurrent or refractory solid tumors have not yet been reported (NCT02564198). Unfortunately, the incorporation 
of anti-VEGF(R) antibodies into the treatment of pediatric solid tumors has been unsuccessful to date and it remains to be seen whether tumor vasculature can be successfully targeted in other ways.

\subsection{Novel Targets Under Active Investigation (Arranged Alphabetically)}

\subsubsection{CD30}

Brentuximab, an anti-CD30 monoclonal antibody, was used to treat seven adult patients ( 5 of which were young adults under the age of 40) with CD30-positive relapsed or refractory germ cell, Leydig cell, and Sertoli cell tumors and demonstrated an objective response in two of the seven (29\%) [51]. Additional study is required.

\subsubsection{CD47}

The clinical therapeutic potential of targeting tumor-associated macrophages (TAMs) was recently shown by inhibition of the macrophage 'checkpoint' SIRP $\alpha$ by interfering with binding to its ligand, CD47, which is often expressed on cancer cells including solid tumors [52]. Hu5F9-G4 (magrolimab) is a humanized monoclonal antibody that binds to CD47 and blocks its anti-phagocytic 'do not eat me' signal, leading to tumor cell phagocytosis; we previously summarized its expression in pediatric histologies [23]. We look forward to several planned phase I clinical trials of Hu5F9-G4 alone or in combination with anti-GD2 therapy for children with advanced solid tumors (NCT04525014, NCT04751383).

\subsubsection{Glycoprotein Non-Metastatic B (gpNMB)}

gpNMB is a type I transmembrane glycoprotein that is overexpressed in malignancy. A phase II trial of glembatumumab vedotin (anti-gpNMB conjugated to monomethyl auristatin E or MMAE) in 22 patients aged $12-50$ years with relapsed or refractory osteosarcoma unfortunately had one grade 5 toxicity possibly related to glembatumumab vedotin, and there was only one PR (4.5\%), so the agent will not proceed to phase II [53]. This agent has been de-prioritized.

\subsubsection{Insulin-Like Growth Factor Receptor 1 (IGF-1R)}

Insulin-like growth factor signaling activation promotes cancer proliferation, invasion, and metastasis but has proven difficult to therapeutically target. A phase III trial was conducted in children with newly diagnosed metastatic Ewing sarcoma (COG AEWS1221) including 148 patients with standard chemotherapy and 151 with standard chemotherapy plus ganitumab, a monoclonal antibody against IGF$1 \mathrm{R}$. Results demonstrated potentially increased toxicity (increased pneumonitis post-radiation) without a survival benefit [54]. Recently, dalotuzumab, another monoclonal antibody against IGF-1R, was studied in combination with ridaforolimus, an mTOR inhibitor, in a phase I study of pediatric patients with advanced solid tumors. Twenty-four patients were treated with a single DLT of stomatitis and one out of six (16.6\%) patients with Ewing sarcoma had a PR [55]. The successful incorporation of IGF-1R antibodies into Ewing sarcoma therapy will require additional research.

\subsubsection{L1-Cell Adhesion Molecule (L1-CAM)}

CAR-T cells against L1-CAM (CD171), a transmembrane glycoprotein overexpressed in cancer, were tested in 22 children with recurrent/refractory high-risk neuroblastoma but showed no objective responses; two patients with early progressive disease were alive at time of publication without subsequent therapy [56]. Increasing CAR dose and CAR generation (third over second) resulted in improved CAR persistence and may deserve additional study.

\subsubsection{Receptor Activator of NFKB Ligand (RANKL)}

Denosumab is a monoclonal antibody that binds RANKL, which is expressed on osteoclasts and may be important in pulmonary metastatic spread of osteosarcoma [57]. This agent was tested in COG trial AOST1321 and demonstrated no efficacy in patients with relapsed osteosarcoma; the data were analyzed in aggregate with two other trials of novel therapies (eribulin and glembatumumab) and those 57 patients were found to have a 4-month EFS of 7\% [58]. This agent may be de-prioritized.

\subsubsection{Semaphorin SEMA4D}

Semaphorins regulate immune responses to tumors and angiogenesis and are overexpressed on solid tumors, including osteosarcoma; pepinemab is a monoclonal antibody against the semaphorin SEMA4D. A phase I/II trial (COG ADVL1614) in patients aged 1-30 years with relapsed or refractory solid tumors treated 16 evaluable patients and was generally well tolerated, with one patient having a cycle 1 DLT (grade 3 acute kidney injury, arthralgias) and another patient have a late-cycle grade 4 pericardial effusion [59]. Drug activity is still under evaluation.

\subsubsection{Tumor Necrosis Factor-Related Apoptosis-Inducing Ligand (TRAIL)}

TRAIL activation can lead to cell death; lexatumumab (HGS-ETR2) is a TRAIL-R2 agonist. A phase I trial in patients aged 1-30 years with relapsed or refractory solid tumors treated 24 patients with a single agent. The trial 
showed no objective responses, but there is a report of a patient with osteosarcoma who experienced resolution of clinical symptoms and PET activity [60]. Further study is needed.

\subsubsection{Other}

Other relevant targets currently in clinical trial, awaiting trial open, or pending reporting of results are listed in Table 1 (including $\alpha 4$-integrin, CD40, CD73, etc.).

\section{Non-Targeted Therapies}

\subsection{Oncolytic Viruses}

Oncolytic viruses are live, replicating viruses that infect and kill tumor cells, induce an antitumor immune response, and are safe to administer to people. In the 6 years since talimogene laherparepvec (T-vec, Imlygic) was approved by the FDA for melanoma in 2015, there has not been another FDA approval, either of T-VEC in a second indication or of another virus. However, it remains an active area of investigation, with over 200 ongoing clinical trials of different viruses spanning numerous types of viruses in combination with a variety of other therapies (recently reviewed here [61]). Poor efficacy is thought to be due to a variety of factors including antiviral immune responses, the immunosuppressive tumor microenvironment, and low delivery of systemically administered virus to tumor sites. Alternative delivery methods to circumvent systemic antiviral immunity are now being pursued. For example, a phase II study evaluated the safety and efficacy of autologous mesenchymal stem cells carrying an oncolytic adenovirus in patients with relapsed/refractory solid tumors, including nine pediatric patients, and demonstrated good safety but no objective responses [62].

Perhaps the most promising area for oncolytic viruses in pediatrics is brain tumors, given that the tumor is usually localized and, despite the inherent challenges, can be injected locally. Friedman and colleagues recently demonstrated feasibility of injecting pediatric patients with an oncolytic herpes simplex virus, G207, and found most patients appeared to benefit with prolonged stable disease or tumor shrinkage [63]. The virus appeared to act mainly as an immunostimulant, recruiting $\mathrm{T}$ cells into the tumor and requiring significant time to elicit tumor responses. Other ongoing oncolytic virus studies in pediatric patients with brain tumors include those based on poliovirus, measles virus, and adenovirus (NCT03043391, NCT02962167, NCT03178032).

\subsection{Vaccines}

Therapeutic cancer vaccines for pediatric malignancies were recently summarized [64].

\subsection{Immunomodulatory Drugs}

In the pathway towards drug discovery, some agents are found to have unforeseen immune effects. Pomalidomide is one such agent where the exact molecular mechanism of antitumor activity is unknown but may broadly suppress various cytokines important for tumor growth while increasing cytokines important for T- and NK-cell tumor suppression [65]. The Pediatric Brain Tumor Consortium (PBTC) recently conducted a phase I trial of pomalidomide in 25 evaluable children with recurrent, refractory, and progressive brain tumors and demonstrated four DLTs in three patients, including grade 3 diarrhea, grade 3 thrombocytopenia, grade 3 lung infection, and grade 4 neutropenia [66]. There was one patient with anaplastic pleomorphic xanthoastrocytoma of four anaplastic astrocytomas (25\%) who demonstrated a sustained PR.

\section{Prioritization}

While very few immunotherapies have proven themselves in these early days as fully established front-line therapies for pediatric solid tumors, the notable exceptions include antiGD2 therapy for neuroblastoma and checkpoint inhibition for melanoma, renal cell carcinoma, and solid tumors with high tumor mutational burden (among other entities outlined here [67]). Biomarkers and assessment of the success of these approaches continues to be a work in progress. Also, it is unclear why these validated antibodies have not yet been converted to antibody-drug conjugates given the success of that class of agents in leukemias/lymphomas and some adult solid tumors [68]. We are not aware of data suggesting such conjugation might interfere with their Fc receptor binding or that there are low rates of receptor internalization upon engagement.

In our opinion, combination trials featuring immunotherapeutic agents plus or minus standard chemotherapeutic agents are the most promising options moving forward. We are encouraged most by the increasing volume of work done on well-known immunologic targets such as PD-1, GD2, B7-H3, HER2, and CD47, but anticipate identification of novel tumor antigens, several of the most promising of which are detailed in this review. It is too early to know which of these will prove to be both safe and efficacious in different diseases and contexts. Prioritization for further development should take into consideration not only the 
strength of preclinical and early clinical data, but also the likelihood and expected timeline of clinical trials as well as the prospect of industry engagement if the target is largely restricted to pediatric (rare) diseases. Some groups such as ACCELERATE have begun having such discussions and even published their rationale for the development of immunotherapies in pediatric patients with cancers and how regulatory barriers may be overcome [69-71]. These documents emphasize the importance of combination trials and encourage the use of checkpoint inhibitors with other immunotherapies, especially in (solid) tumors with hypermutation (which is the only identified predictive biomarker to date). In particular, they emphasize the combination of checkpoint inhibitors with CAR-T cells and engineered antibody-based proteins, also known as synthetic immunotherapies [69]. In one discussion dedicated to epigenetic modifiers, they provide theoretical insight into how such agents may be combined with immunotherapy, potentially by upregulating MHC-I expression [71].

Regarding non-targeted agents, the field is too underdeveloped to make any judgements about prioritization. Notably, the clinical successes of mRNA vaccines delivered by liposomal nanoparticles for Sars-CoV-2 has recently increased enthusiasm for mRNA vaccines for cancer, but it is unclear if cancer neoantigens will be sufficiently immunogenic to break tolerance with that approach.

\section{Conclusion}

As with other aspects of cancer, the old adage appears to be holding true with immunotherapies: children are not young adults, and neither are their cancers. With rare case exceptions, the success of checkpoint inhibition in adult cancers has not been realized in pediatrics, likely due to generally low mutational burdens. Yet there has been great progress in leveraging the immune system to attack cancers by recognizing normal proteins or antigens (e.g., CD19 and GD2) that can be clinically tolerated. As evidenced in our review, much work is underway to evaluate the utility of numerous other immunologic targets. The lack of other FDA approvals beyond anti-CD19 and anti-GD2 therapies in pediatric cancers (and the restricted approval of checkpoint inhibitors for mismatch repair patients) exemplifies the challenges that remain.

With all the progress in just a few years, the era of pediatric immunotherapies appears to be 'hitting the gas.' But in order to truly accelerate, we must highlight the importance of counteracting the immunosuppressive microenvironment and 'releasing the brakes.' It is becoming increasingly clear from pre-clinical and human tissue studies that pediatric cancers often harbor numerous immunosuppressive cells or factors (Tregs, myeloid-derived supressor cells, M2-like macrophages, PD-L1, hypoxia, arginine depletion, adenosine, IL-10, etc.). To realize the full potential of immunotherapies, we will likely need to co-develop strategies or treatments to 'release' all of these different 'brakes' in the tumor microenvironment, allowing immunotherapies to travel that much farther.

\section{Declarations}

Funding National Cancer Institute Cancer Moonshot Award U54CA232561-01A1 (TPC).

Conflict of interest A. Gupta and T.P. Cripe declare that they have no conflicts of interest that might be relevant to the contents of this manuscript.

Ethics approval Not applicable.

Consent to participate Not applicable.

Consent for publication Not applicable.

Availability of data and material Not applicable.

Code availability Not applicable.

Author contributions A. Gupta and T.P. Cripe contributed equally to the development and writing of this manuscript.

\section{References}

1. Wedekind MF, Denton NL, Chen CY, Cripe TP. Pediatric cancer immunotherapy: opportunities and challenges. Paediatr Drugs. 2018;5:395-408. https://doi.org/10.1007/s40272-018-0297-x.

2. Pacenta HL, Laetsch TW, John S. CD19 CAR T cells for the treatment of pediatric pre-B cell acute lymphoblastic leukemia. Paediatr Drugs. 2020;1:1-11. https://doi.org/10.1007/ s40272-019-00370-6.

3. Jasinski S, De Los Reyes FA, Yametti GC, Pierro J, Raetz E, Carroll WL. Immunotherapy in pediatric B-cell acute lymphoblastic leukemia: advances and ongoing challenges. Paediatr Drugs. 2020;5:485-99. https://doi.org/10.1007/s40272-020-00413-3.

4. Rodrigues MN, Natoli M, Zippelius A, Laubli H. Tumor-associated carbohydrates and immunomodulatory lectins as targets for cancer immunotherapy. J Immunother Cancer. 2020. https://doi. org/10.1136/jitc-2020-001222.

5. Chan CJ, Smyth MJ, Martinet L. Molecular mechanisms of natural killer cell activation in response to cellular stress. Cell Death Differ. 2014;1:5-14. https://doi.org/10.1038/cdd.2013.26.

6. First Tissue-Agnostic Drug Approval Issued. Cancer Discov. 2017;7:656. https://doi.org/10.1158/2159-8290.Cd-nb2017-078.

7. Davis KL, Fox E, Merchant MS, Reid JM, Kudgus RA, Liu X, et al. Nivolumab in children and young adults with relapsed or refractory solid tumours or lymphoma (ADVL1412): a multicentre, open-label, single-arm, phase 1-2 trial. Lancet Oncol. 2020;4:541-50. https://doi.org/10.1016/s1470-2045(20)30023-1.

8. Geoerger B, Kang HJ, Yalon-Oren M, Marshall LV, Vezina C, Pappo A, et al. Pembrolizumab in paediatric patients with advanced melanoma or a PD-L1-positive, advanced, relapsed, or 
refractory solid tumour or lymphoma (KEYNOTE-051): interim analysis of an open-label, single-arm, phase 1-2 trial. Lancet Oncol. 2020;1:121-33. https://doi.org/10.1016/s1470-2045(19) 30671-0.

9. Tawbi HA, Burgess M, Bolejack V, Van Tine BA, Schuetze SM, $\mathrm{Hu}$ J, et al. Pembrolizumab in advanced soft-tissue sarcoma and bone sarcoma (SARC028): a multicentre, two-cohort, singlearm, open-label, phase 2 trial. Lancet Oncol. 2017;11:1493-501. https://doi.org/10.1016/s1470-2045(17)30624-1.

10. Naqash AR, O'Sullivan GHC, Moore N, Sharon E, Takebe N, Fino $\mathrm{KK}$, et al. Phase II study of atezolizumab in advanced alveolar soft part sarcoma (ASPS). J Clin Oncol. 2021;15:11519-11519. https://doi.org/10.1200/JCO.2021.39.15_suppl.11519.

11. Gordon EM, Chua-Alcala VS, Kim K, Baby R, Angel N, Quon D, et al. A phase I/II investigation of nivolumab and ABI-009 (nabsirolimus) in advanced undifferentiated pleomorphic sarcoma (UPS), liposarcoma (LPS), chondrosarcoma (CS), osteosarcoma (OS), and Ewing sarcoma: Preliminary efficacy and safety results. J Clin Oncol. 2019;15:11057-11057. https://doi.org/10.1200/JCO. 2019.37.15_suppl.11057.

12. Pasqualini C, Rubino J, Brard C, Cassard L, André N, Rondof $\mathrm{W}$, et al. Phase II and biomarker study of programmed cell death protein 1 inhibitor nivolumab and metronomic cyclophosphamide in paediatric relapsed/refractory solid tumours: Arm G of AcSéESMART, a trial of the European Innovative Therapies for Children With Cancer Consortium. Eur J Cancer. 2021. https://doi. org/10.1016/j.ejca.2021.03.032.

13. Wienke J, Dierselhuis MP, Tytgat GAM, Kunkele A, Nierkens $\mathrm{S}$, Molenaar JJ. The immune landscape of neuroblastoma: challenges and opportunities for novel therapeutic strategies in pediatric oncology. Eur J Cancer. 2021. https://doi.org/10.1016/j.ejca. 2020.11.014

14. Mardanpour K, Rahbar M, Mardanpour S, Mardanpour N, Rezaei M. CD8+ T-cell lymphocytes infiltration predict clinical outcomes in Wilms' tumor. Tumour Biol. 2020;12:1010428320975976. https://doi.org/10.1177/1010428320975976.

15. Boldrini R, De Pasquale MD, Melaiu O, Chierici M, Jurman G, Benedetti MC, et al. Tumor-infiltrating T cells and PD-L1 expression in childhood malignant extracranial germ-cell tumors. Oncoimmunology. 2019;2:e1542245. https://doi.org/10.1080/21624 02x.2018.1542245.

16. Plant AS, Koyama S, Sinai C, Solomon IH, Griffin GK, Ligon KL, et al. Immunophenotyping of pediatric brain tumors: correlating immune infiltrate with histology, mutational load, and survival and assessing clonal T cell response. J Neurooncol. 2018;2:26978. https://doi.org/10.1007/s11060-017-2737-9.

17. Palmerini E, Agostinelli C, Picci P, Pileri S, Marafioti T, Lollini PL, et al. Tumoral immune-infiltrate (IF), PD-L1 expression and role of CD8/TIA-1 lymphocytes in localized osteosarcoma patients treated within protocol ISG-OS1. Oncotarget. 2017;67:111836-46. https://doi.org/10.18632/oncotarget.22912.

18. Cornel AM, Mimpen IL, Nierkens S. MHC class I downregulation in cancer: underlying mechanisms and potential targets for cancer immunotherapy. Cancers (Basel). 2020. https://doi.org/10.3390/ cancers 12071760 .

19. Ladenstein R, Pötschger U, Valteau-Couanet D, Luksch R, Castel V, Yaniv I, et al. Interleukin 2 with anti-GD2 antibody ch14.18/ $\mathrm{CHO}$ (dinutuximab beta) in patients with high-risk neuroblastoma (HR-NBL1/SIOPEN): a multicentre, randomised, phase 3 trial. Lancet Oncol. 2018;12:1617-29. https://doi.org/10.1016/s14702045(18)30578-3.

20. Morandi F, Sabatini F, Podesta M, Airoldi I. Immunotherapeutic strategies for neuroblastoma: present, past and future. Vaccines (Basel). 2021. https://doi.org/10.3390/vaccines9010043.
21. Dobrenkov K, Ostrovnaya I, Cheung IY, Feng Y, Cheung N-KV. GD2/GD3 expression: companion diagnostic for ganglioside-targeted immunotherapy against pediatric solid tumors. J Clin Oncol. 2016;15:10567-10567. https://doi.org/10.1200/JCO.2016.34.15_ suppl.10567.

22. Hingorani P, Krailo MD, Buxton A, Hutson PR, Davis J, Janeway $\mathrm{KA}$, et al. Phase II study of antidisialoganglioside antibody, dinutuximab, in combination with GM-CSF in patients with recurrent osteosarcoma (AOST1421): a report from the Children's Oncology Group. J Clin Oncol. 2020;15:10508-10508. https://doi.org/ 10.1200/JCO.2020.38.15_suppl.10508.

23. Gupta A, Taslim C, Tullius BP, Cripe TP. Therapeutic modulation of the CD47-SIRP $\alpha$ axis in the pediatric tumor microenvironment: working up an appetite. Cancer Drug Resistance. 2020;3:550-62. https://doi.org/10.20517/cdr.2020.12.

24. Nazha B, Inal C, Owonikoko TK. Disialoganglioside GD2 expression in solid tumors and role as a target for cancer therapy. Front Oncol. 2020. https://doi.org/10.3389/fonc.2020.01000.

25. Heczey A, Courtney AN, Montalbano A, Robinson S, Liu K, Li M, et al. Anti-GD2 CAR-NKT cells in patients with relapsed or refractory neuroblastoma: an interim analysis. Nat Med. 2020;11:1686-90. https://doi.org/10.1038/s41591-020-1074-2.

26. Heczey A, Courtney AN, Liu K, Li M, Ghatwai N, Dakhova O, et al. Natural killer T cells expressing a GD2-CAR and IL-15 are safe and can induce complete remission in children with relapsed neuroblastoma - a first-in-human, Phase 1 Trial. Mol Ther. 2021;4(Supplement 1):102-3. https://doi.org/10.1016/j.ymthe. 2021.04.019.

27. Straathof K, Flutter B, Wallace R, Thomas S, Cheung G, Collura A, et al. Abstract CT145: a Cancer Research UK phase I trial of anti-GD2 chimeric antigen receptor (CAR) transduced T-cells (1RG-CART) in patients with relapsed or refractory neuroblastoma. Cancer Res. 2018;13:CT145-CT145. https://doi.org/10. 1158/1538-7445.Am2018-ct145.

28. Yang L, Ma X, Liu Y-C, Zhao W, Yu L, Qin M, et al. Chimeric antigen receptor 4SCAR-GD2-modified T cells targeting highrisk and recurrent neuroblastoma: a Phase II multi-center trial in China. Blood. 2017;1:3335-3335. https://doi.org/10.1182/blood. V130.Suppl_1.3335.3335.

29. Richards RM, Sotillo E, Majzner RG. CAR T Cell Therapy for Neuroblastoma. Front Immunol. 2018. https://doi.org/10.3389/ fimmu.2018.02380.

30. Ehlert K, Hansjuergens I, Zinke A, Otto S, Siebert N, Henze G, et al. Nivolumab and dinutuximab beta in two patients with refractory neuroblastoma. J Immunother Cancer. 2020. https://doi.org/ 10.1136/jitc-2020-000540.

31. Heczey A, Louis CU, Savoldo B, Dakhova O, Durett A, Grilley B, et al. CAR T cells administered in combination with lymphodepletion and PD-1 inhibition to patients with neuroblastoma. Mol Ther. 2017;9:2214-24. https://doi.org/10.1016/j.ymthe.2017.05. 012.

32. Federico SM, McCarville MB, Shulkin BL, Sondel PM, Hank JA, Hutson P, et al. A Pilot Trial of Humanized Anti-GD2 Monoclonal Antibody (hu14.18K322A) with Chemotherapy and Natural Killer Cells in Children with Recurrent/Refractory Neuroblastoma. Clin Cancer Res. 2017;21:6441-9. https://doi.org/10.1158/1078-0432. CCR-17-0379.

33. Modak S, Le Luduec JB, Cheung IY, Goldman DA, Ostrovnaya I, Doubrovina E, et al. Adoptive immunotherapy with haploidentical natural killer cells and Anti-GD2 monoclonal antibody m3F8 for resistant neuroblastoma: results of a phase I study. Oncoimmunology. 2018;8:e1461305. https://doi.org/10.1080/2162402X.2018. 1461305.

34. Choi YB, Son MH, Cho HW, Ma Y, Lee JW, Kang ES, et al. Safety and immune cell kinetics after donor natural killer cell infusion 
following haploidentical stem cell transplantation in children with recurrent neuroblastoma. PLoS ONE. 2019;12:e0225998. https:// doi.org/10.1371/journal.pone.0225998.

35. Talleur AC, Triplett BM, Federico S, Mamcarz E, Janssen W, Wu $\mathrm{J}$, et al. Consolidation Therapy for Newly Diagnosed Pediatric Patients with High-Risk Neuroblastoma Using Busulfan/Melphalan, Autologous Hematopoietic Cell Transplantation, Anti-GD2 Antibody, Granulocyte-Macrophage Colony-Stimulating Factor, Interleukin-2, and Haploidentical Natural Killer Cells. Biol Blood Marrow Transpl. 2017;11:1910-7. https://doi.org/10.1016/j.bbmt. 2017.07.011.

36. Souweidane MM, Kramer K, Pandit-Taskar N, Zhou Z, Haque $\mathrm{S}$, Zanzonico $\mathrm{P}$, et al. Convection-enhanced delivery for diffuse intrinsic pontine glioma: a single-centre, dose-escalation, phase 1 trial. Lancet Oncol. 2018;8:1040-50. https://doi.org/10.1016/ s1470-2045(18)30322-x.

37. Souweidane MM, Kramer K, Pandit-Taskar N, Haque S, Zanzonico P, Carrasquillo JA, et al. Phase 1 dose-escalation trial using convection-enhanced delivery of radiolabeled monoclonal antibody for diffuse intrinsic pontine glioma following external radiation therapy. J Clin Oncol. 2021;15:2010-2010. https://doi. org/10.1200/JCO.2021.39.15_suppl.2010.

38. Modak S, Zanzonico P, Grkovski M, Slotkin EK, Carrasquillo JA, Lyashchenko SK, et al. B7H3-directed intraperitoneal radioimmunotherapy with radioiodinated omburtamab for desmoplastic small round cell tumor and other peritoneal tumors: results of a Phase I study. J Clin Oncol. 2020;36:4283-91. https://doi.org/10. 1200/jco.20.01974.

39. Modak S, Saltsman J, Pandit-Taskar N, Slotkin EK, Heaton T, Gerstle J et al (2019) Whole Abdominopelvic Radiotherapy and Radioimmunotherapy After Complete Resection of Desmoplastic Small Round Cell Tumor (DSRCT): Major Impact on Survival. CTOS Annual Meeting

40. Kramer K, Kushner BH, Modak S, Pandit-Taskar N, Tomlinson $\mathrm{U}$, Wolden SL, et al. A curative approach to central nervous system metastases of neuroblastoma. J Clin Oncol. 2017;15:1054510545. https://doi.org/10.1200/JCO.2017.35.15_suppl.10545.

41. The Lancet O CAR T-cell therapy for solid tumours. The Lancet Oncology. 2021;7:893. https://doi.org/10.1016/S1470-2045(21) 00353-3.

42. Navai SA, Derenzo C, Joseph S, Sanber K, Byrd T, Zhang H, et al. Abstract LB-147: Administration of HER2-CAR T cells after lymphodepletion safely improves $\mathrm{T}$ cell expansion and induces clinical responses in patients with advanced sarcomas. Cancer Res. 2019;13:LB-147-LB-147. https://doi.org/10.1158/15387445.Am2019-lb-147.

43. Hegde M, Joseph SK, Pashankar F, DeRenzo C, Sanber K, Navai $\mathrm{S}$, et al. Tumor response and endogenous immune reactivity after administration of HER2 CAR T cells in a child with metastatic rhabdomyosarcoma. Nat Commun. 2020;1:3549. https://doi.org/ 10.1038/s41467-020-17175-8.

44. Ahmed N, Brawley V, Hegde M, Bielamowicz K, Kalra M, Landi $\mathrm{D}$, et al. HER2-specific chimeric antigen receptor-modified virusspecific $\mathrm{T}$ cells for progressive glioblastoma: a phase 1 dose-escalation trial. JAMA Oncol. 2017;8:1094-101. https://doi.org/10. 1001/jamaoncol.2017.0184.

45. Slotkin EK, Magnan HD, Meyers PA, Chou AJ, Ambati SR, Wexler LH. Off-label use of bevacizumab in relapsed and refractory pediatric sarcoma patients: the Memorial Sloan Kettering Cancer Center Experience. J Clin Oncol. 2016;15:10569-10569. https:// doi.org/10.1200/JCO.2016.34.15_suppl.10569.

46. Santana VM, Sahr N, Tatevossian RG, Jia S, Campagne O, Sykes A, et al. A phase 1 trial of everolimus and bevacizumab in children with recurrent solid tumors. Cancer. 2020;8:1749-57. https://doi. org/10.1002/cncr.32722.
47. Federico SM, Caldwell KJ, McCarville MB, Daryani VM, Stewart $\mathrm{CF}$, Mao S, et al. Phase I expansion cohort to evaluate the combination of bevacizumab, sorafenib and low-dose cyclophosphamide in children and young adults with refractory or recurrent solid tumours. Eur J Cancer. 2020. https://doi.org/10.1016/j.ejca.2020. 03.010.

48. Ferrari A, Merks JHM, Chisholm JC, Orbach D, Brennan B, Gallego $S$, et al. Outcomes of metastatic non-rhabdomyosarcoma soft tissue sarcomas (NRSTS) treated within the BERNIE study: a randomised, phase II study evaluating the addition of bevacizumab to chemotherapy. Eur J Cancer. 2020. https://doi.org/10.1016/j.ejca. 2020.01.029.

49. Chisholm JC, Merks JHM, Casanova M, Bisogno G, Orbach D, Gentet JC, et al. Open-label, multicentre, randomised, phase II study of the EpSSG and the ITCC evaluating the addition of bevacizumab to chemotherapy in childhood and adolescent patients with metastatic soft tissue sarcoma (the BERNIE study). Eur J Cancer. 2017. https://doi.org/10.1016/j.ejca.2017.06.015.

50. Navid F, Santana VM, Neel M, McCarville MB, Shulkin BL, $\mathrm{Wu}$ J, et al. A phase II trial evaluating the feasibility of adding bevacizumab to standard osteosarcoma therapy. Int J Cancer. 2017;7:1469-77. https://doi.org/10.1002/ijc.30841.

51. Albany C, Einhorn L, Garbo L, Boyd T, Josephson N, Feldman DR. Treatment of CD30-expressing germ cell tumors and sex cord stromal tumors with brentuximab vedotin: identification and report of seven cases. Oncologist. 2018;3:316-23. https://doi.org/ 10.1634/theoncologist.2017-0544.

52. Willingham SB, Volkmer JP, Gentles AJ, Sahoo D, Dalerba $\mathrm{P}$, Mitra SS, et al. The CD47-signal regulatory protein alpha (SIRPa) interaction is a therapeutic target for human solid tumors. Proc Natl Acad Sci USA. 2012;17:6662-7. https://doi. org/10.1073/pnas.1121623109.

53. Kopp LM, Malempati S, Krailo M, Gao Y, Buxton A, Weigel BJ, et al. Phase II trial of the glycoprotein non-metastatic B-targeted antibody-drug conjugate, glembatumumab vedotin (CDX011), in recurrent osteosarcoma AOST1521: a report from the Children's Oncology Group. Eur J Cancer. 2019. https://doi.org/ 10.1016/j.ejca.2019.08.015.

54. DuBois SG, Glade-Bender J, Buxton A, Reed DR, Janeway KA, Krailo $M$ et al Randomized Phase 3 Trial of Ganitumab Added to Interval Compressed Chemotherapy for Patients With Newly Diagnosed Metastatic Ewing Sarcoma: A Report From the Children's Oncology Group (COG). CTOS Annual Meeting. 2019

55. Frappaz D, Federico SM, Pearson AD, Gore L, Macy ME, DuBois SG, et al. Phase 1 study of dalotuzumab monotherapy and ridaforolimus-dalotuzumab combination therapy in paediatric patients with advanced solid tumours. Eur J Cancer. 2016. https://doi.org/10.1016/j.ejca.2016.03.084.

56. Pinto N, Kuenkele A, Gardner R, Finney O, Brakke H, Brown C, ENCIT-01: A Phase 1 Study of Autologous T-Cells Lentivirally Transduced to Express CD171-Specific chimeric antigen receptors for recurrent/refractory high-risk neuroblastoma. Adv Neuroblastoma Res. 2018

57. Navet B, Ando K, Vargas-Franco JW, Brion R, Amiaud J, Mori $\mathrm{K}$, et al. The intrinsic and extrinsic implications of RANKL/ RANK signaling in osteosarcoma: from tumor initiation to lung metastases. Cancers (Basel). 2018. https://doi.org/10.3390/ cancers 10110398.

58. Rao S, Han R, Krailo MD, Buxton A, Hingorani P, Chou AJ, et al. Outcome of patients with recurrent/refractory osteosarcoma enrolled in three recent phase II trials: a report from the Children's Oncology Group. J Clin Oncol. 2021;15:1153011530. https://doi.org/10.1200/JCO.2021.39.15_suppl.11530.

59. Greengard EG, Williams RL, Liu X, Militano O, Fisher TL, Evans EE, et al. A phase I/II trial of VX15/2503 in children, adolescents, and young adults with relapsed or refractory solid 
tumors (ADVL1614). J Clin Oncol. 2019;15:e21519-e21519. https://doi.org/10.1200/JCO.2019.37.15_suppl.e21519.

60. Merchant MS, Geller JI, Baird K, Chou AJ, Galli S, Charles A, et al. Phase I trial and pharmacokinetic study of lexatumumab in pediatric patients with solid tumors. J Clin Oncol. 2012;33:4141-7. https://doi.org/10.1200/jco.2012.44.1055.

61. Malogolovkin A, Gasanov N, Egorov A, Weener M, Ivanov R, Karabelsky A. Combinatorial approaches for cancer treatment using oncolytic viruses: projecting the perspectives through clinical trials outcomes. Viruses. 2021. https://doi.org/10.3390/ v13071271.

62. Ruano D, Lopez-Martin JA, Moreno L, Lassaletta A, Bautista F, Andion M, et al. First-in-human, first-in-child trial of autologous MSCs carrying the oncolytic virus icovir-5 in patients with advanced tumors. Mol Ther. 2020;4:1033-42. https://doi.org/10. 1016/j.ymthe.2020.01.019.

63. Friedman GK, Johnston JM, Bag AK, Bernstock JD, Li R, Aban I, et al. Oncolytic HSV-1 G207 immunovirotherapy for pediatric high-grade gliomas. N Engl J Med. 2021;17:1613-22. https:// doi.org/10.1056/NEJMoa2024947.

64. Olsen HE, Lynn GM, Valdes PA, Cerecedo CDL, Ishizuka AS, Arnaout $\mathrm{O}$ et al Therapeutic cancer vaccines for pediatric malignancies: advances, challenges, and emerging technologies. Neurooncol Adv. 2021; 1:27 https://doi.org/10.1093/noajnl/vdab0 27.

65. Chanan-Khan AA, Swaika A, Paulus A, Kumar SK, Mikhael JR, Rajkumar SV, et al. Pomalidomide: the new immunomodulatory agent for the treatment of multiple myeloma. Blood Cancer J. 2013;9:e143. https://doi.org/10.1038/bcj.2013.38.

66. Fangusaro J, Mitchell DA, Kocak M, Robinson GW, Baxter PA, Hwang EI, et al. Phase 1 study of pomalidomide in children with recurrent, refractory, and progressive central nervous system tumors: a pediatric brain tumor consortium trial. Pediatr Blood Cancer. 2021;2:e28756. https://doi.org/10.1002/pbc.28756.

67. Vaddepally RK, Kharel P, Pandey R, Garje R, Chandra AB. Review of indications of FDA-approved immune checkpoint inhibitors per NCCN guidelines with the level of evidence. Cancers (Basel). 2020. https://doi.org/10.3390/cancers12030738.

68. Baah S, Laws M, Rahman KM. Antibody-drug conjugates-A tutorial review. Molecules. 2021. https://doi.org/10.3390/molecules2 6102943.
69. Pearson ADJ, Rossig C, Lesa G, Diede SJ, Weiner S, Anderson J, et al. ACCELERATE and European Medicines Agency Paediatric Strategy Forum for medicinal product development of checkpoint inhibitors for use in combination therapy in paediatric patients. Eur J Cancer. 2020. https://doi.org/10.1016/j.ejca.2019.12.029.

70. Reaman G, Karres D, Ligas F, Lesa G, Casey D, Ehrlich L, et al. Accelerating the Global Development of Pediatric Cancer Drugs: A Call to Coordinate the Submissions of Pediatric Investigation Plans and Pediatric Study Plans to the European Medicines Agency and US Food and Drug Administration. J Clin Oncol. 2020;36:4227-30. https://doi.org/10.1200/jco.20.02152.

71. Pearson AD, Stegmaier K, Bourdeaut F, Reaman G, Heenen D, Meyers ML, et al. Paediatric Strategy Forum for medicinal product development of epigenetic modifiers for children: ACCELERATE in collaboration with the European Medicines Agency with participation of the Food and Drug Administration. Eur J Cancer. 2020. https://doi.org/10.1016/j.ejca.2020.08.014.

72. Mora J, Chan G, Morgenstern D, Nysom K, Bear M, Dalby LW, et al. International, Multicenter Phase II Trial With Humanized Anti-GD2 Monoclonal Antibody Naxitamab for Treatment of Refractory/Relapsed High-Risk Neuroblastoma: Efficacy and Safety Data. Pediatr Blood Cancer. 2020;S4:e28742. https://doi. org/10.1002/pbc.28742.

73. Majzner R, Ramakrishna S, Mochizuki A, Patel S, Chinnasamy H, Yeom $\mathrm{K}$ et al EPCT-14. GD2 CAR T-CELLS MEDIATE CLINICAL ACTIVITY AND MANAGEABLE TOXICITY IN CHILDREN AND YOUNG ADULTS WITH H3K27M-MUTATED DIPG AND SPINAL CORD DMG. Neuro-Oncology. 2021. https://doi.org/10.1093/neuonc/noab090.200.

74. Reilley MJ, Bailey A, Subbiah V, Janku F, Naing A, Falchook G, et al. Phase I clinical trial of combination imatinib and ipilimumab in patients with advanced malignancies. J Immunother Cancer. 2017. https://doi.org/10.1186/s40425-017-0238-1.

75. Butler MO, Sotov V, Saibil S, Bonilla L, Takahashi S, Tanaka S, et al. 4739 - Adoptive T cell therapy with TBI-1301 results in gene-engineered $\mathrm{T}$ cell persistence and anti-tumor responses in patients with NY-ESO-1 expressing solid tumors. Ann Oncol. 2019;5:v475-532. https://doi.org/10.1093/annonc/mdz253. 